together with a determination of the melting points for the resulting semicarbazones. Current spectrographic work further confirms these results. The work is continuing; it is hopod to publish more information on this combustion chamber.

J. M. F. VICkers

Mechanical Enginecring Department,

University of Nebraska,

Lincoln 8, Nebraska. Aug. 5.

"Ranque, G. J., J. Phys, et Rad., 4, 324 (1933).

"Westley, R., "A Bibliography of the Vortex Tubc", College of Aeronautics, Cranfleld, Note No. 9 (1954).

Moore, N. P. W., and Martin, D. G., Fuel, 32, 393 (1953).

\section{Spectra of Flames supported by Active Nitrogen}

WHEN organic substances are introduced into a stream of active nitrogen, a luminous reaction zone is observed, closely resembling the atomic flames given by hydrogen and oxygen. The spectra of these luminous reaction zones have been photographed on 'Ilford Zenith' plates, using a 'Hilger' medium quartz spectrometer and exposure times of 4-6 hr.

Cylinder nitrogen with less than 0.05 per cent of oxygen was activated in a high-frequency electrodeless discharge. Flow rates of $10-15 \mathrm{~cm} .{ }^{3} / \mathrm{sec}$. were used, and the pressure in the apparatus was usually 2-4 mm. of mercury. The organic reactants were introduced into the active nitrogen at a point about $4 \mathrm{~cm}$. beyond the discharge tube, and the luminous zone was viewed through a silica window attached to the end of the reaction tube.

The size and shape of the zone depended largely on the flow rate of the organic reactant. In the present work, the flow rate was adjustod so that the luminous zone was localized on the nitrogen inlet tube, which corresponded to a flow rate of about $0.5 \mathrm{~cm} .{ }^{8} / \mathrm{sec}$. in the case of acetylene. The reaction tube had to be cleaned periodically since most substances used formed a polymer on the walls of the tubo.

The spectra of flames produced by the following substances were photographed :

$\mathrm{C}_{2} \mathrm{H}_{2}, \mathrm{C}_{2} \mathrm{H}_{4}$, cis $-\mathrm{CHCl}=\mathrm{CHCl}, \mathrm{CHCl}=\mathrm{CCl}_{2}, \mathrm{CCl}_{2}=\mathrm{CCl}_{2}$, $\mathrm{CH}_{3} \mathrm{Cl}, \mathrm{CH}_{2} \mathrm{Cl}_{2}, \mathrm{CHCl}_{3}, \mathrm{CCl}_{4}, \mathrm{C}_{2} \mathrm{H}_{6} \mathrm{Cl} ; \mathrm{C}_{2} \mathrm{D}_{2}$

In all cases, the $\mathrm{CN}$-violet system was the most prominent feature of the spectrum, many of the so-called 'tail bands' being present. The CN-red system was also prominent, and appoared to be enhanced by the presence of chlorine in the molecule. All substances containing chlorine gave the CCl-bands in the region of $2790 \mathrm{~A}$., but no bands that might correspond to the unknown molecule $\mathrm{NCl}$ could be found. All molecules containing hydrogen gave the CH-band at $4315 \mathrm{~A}$., due to the transition ${ }^{2} \Delta \rightarrow^{2} \pi$; but the band at $3889 \mathrm{~A}$., due to the transition ${ }^{2} \Sigma \rightarrow{ }^{2} \pi$, appearod to be absent. These molecules also gave the NH-hoads at $3360 \mathrm{~A}$. and $3370 \mathrm{~A}$., the rotational fine structure being visible on some plates. The $\mathrm{CH}$ and NH-bands given by chloroform were very weak, in contrast to the very strong bands given by methylen $\Theta$ dichloride. Because of the strength of the CN-red system, it was often difficult to decide whether the $C_{2}$ Swan-bands were present or not. They were certainly given by acetylene and ethylene, but were not given by carbon tetrachloride. The nitrous oxide $\beta$ - and $\gamma$-systems were occasionally present fuintly, espocially in the case of carbon tetrachloride and tetrachlorethylene.
In addition to the above bands, a complex group of heads was observed in the region of $3290 \mathrm{~A}$., degraded to the violet, with all molecules except carbon tetrachloride and tetrachlorethylene, and possibly chloroform. The intensity of these bands correlates quite well with the intensity of the CH. and NH.bands, and because of this and their complex structure, it was thought that the bands were due to hydrogen cyanide, which is known to be the main product in the reaction of hydrocarbons with active nitrogen ${ }^{1}$. Consequently, deuteroacetylene $\left(\mathrm{C}_{2} \mathrm{D}_{2}\right)$ was prepared by the action of heavy water on purified calcium carbide, giving a product of 97.5 per cent purity, and the spectrum of the flame produced by passing this into active nitrogen was photographed. The spectrum of an iron hollow-cathode lamp was used as a comparison spectrum. A comparison of this plate with one given by methyleno dichloride showed that the structure of the unknown bands was identical, and that the expected isotopic shift was less than $4 \mathrm{~cm}^{-1}$. It therefore seems unlikely that the bands can be attributed to an emitter containing hydrogen, such as hydrogen cyanido.

The fact that the bands are given only by molecules which contain hydrogen may be due to some kinetic requirement, but the bands are not given when molecular hydrogen and carbon tetrachloride are introduced into active nitrogen. It is hoped that further work now in progress will help to clarify the problem.

This work was carried out as part of Contract AF 61(514)-1117 with the A.R.D.C. of the United States Air Foree. We thank H. W. Thompson for the loan of a spectrograph.

K. R. JENnINGS

J. W. LINNETT

Inorganic Chemistry Laboratory,

Oxford. July 30.

1 Livans, H. G. V., Freeman, G. R., and Winkler, C. A., Can. J. Chem., 34, $1271(1956)$.

\section{Production of Vibrationally Excited Oxygen Molecules in the Flash Photolysis of Ozone}

Previous work on the photolysis of ozone has led to the conclusion that a simple atom-chain mechanism is incapable of explaining the experimental facts. The existence of an energy chain involving excited oxygon molecules has gained wide acceptance due to the work of Schumacher ${ }^{1}$, Heidt and Forbes ${ }^{2}$, and Ritchie $^{3}$. The nature of the excitation involved in the energy chain was not known, however, most workers assuming it to be the ${ }^{1} \sum_{g}^{+}$state of the oxygen ${ }^{4}$. Experiments on the flash photolysis of ozono ${ }^{5}$ have enabled us to show that the excitation is purely vibrational, the oxygen being formod in the ground electronic state with up to 16 quanta of vibrational energy, by reaction (1) which is exothermic to the extent of $93 \mathrm{kcal} / \mathrm{mole}$ for $\mathrm{O}\left({ }^{3} P\right)$ and $138 \mathrm{kcal} . / \mathrm{mole}$ for $O\left({ }^{1} D\right)$.

$$
\mathrm{O}+\mathrm{O}_{3} \rightarrow \mathrm{O}^{*}{ }_{2}+\mathrm{O}_{2}
$$

After allowing for the observed vibrational excita. tion and considering that spin conservation will be effective, the second oxygen molecule should be produced in the ${ }^{1} \Delta g$ state according to

$$
\mathrm{O}\left({ }^{3} P\right)+\mathrm{O}_{3}\left({ }^{1} A\right) \rightarrow \mathrm{O}_{2}{ }^{*}\left({ }^{3} \Sigma_{g}^{-}\right)+\mathrm{O}_{2}\left({ }^{1} \Delta g\right)
$$

which would reduce the amount of energy available 